\title{
Novel wearable antenna systems for high datarate mobile communication in healthcare
}

\author{
Hendrik Rogier, Sam Agneessens, Thijs Castel, Sam Lemey, Frederick Declercq, \\ Peter Vanveerdeghem, Patrick Van Torre, Luigi Vallozzi and Wout Joseph \\ Dept. of Information Technology, iMinds/Ghent University \\ Sint-Pietersnieuwstraat, 9000 Gent, Belgium \\ Email: hendrik.rogier@intec.ugent.be
}

\begin{abstract}
In critical healthcare applications, there is a need for reliable wideband mobile communication links, implemented by portable units with sufficient autonomy. We present the latest generation wearable antenna systems for invisible and comfortable integration in patients' or caregivers' garments. These active textile modules boast excellent performance and reliability, thanks to innovative antenna topologies, leveraged by the application of substrate integrated waveguide technology, pervasive integration of electronics and energy harvesters, and the application of multiantenna processing techniques. Applications range from mobile communication links between caregivers and a coordination centre during interventions, over wireless sensor systems for patient monitoring, to relaying videos streams between a wireless endoscopy capsule and a remote control station.
\end{abstract}

Keywords-textile antennas, wearable electronics, body-centric communication.

\section{INTRODUCTION}

Wearable personal monitoring, sensing, communicating and positioning systems realize large added value and important benefits in modern healthcare by implementing realtime continuous surveillance of victims and of rescue workers during interventions and by remotely monitoring patients, to reduce the time spent in hospitals and to improve the life of patients or elderly in their home environment. In this context, smart textiles interactive fabrics (SFIT) implementations seem highly attractive, as they have the potential of combining excellent performance with wearer comfort. Yet, although heavily researched, up to now very few commercial systems based on smart textiles are found on the market. Indeed, reliability, maintenance and cost issues have impeded their breakthrough. Fortunately, recently a lot of work has been performed to overcome the aforementioned problems [1]-[3].

We present our latest generation wearable antenna systems for reliable and energy-efficient operation in healthcare applications. Following novel features were implemented to guarantee stable operation, robustness and user comfort:

- Substrate Integrated Waveguide (SIW) technology is applied to textile materials [4]-[6] to fabricate novel compact textile antennas with excellent isolation from the human body and, hence, excellent performance in a body-centric context.

- Pervasive integration of active electronics [7], [8] and energy harvesters on the textile antenna platform, without affecting the antenna performance. This improves the reliability and autonomy of the active antenna module by avoiding long RF interconnects. The hybrid fabrication process, combining textile materials with ultrathin flexible polyimide substrates, guarantees flexibility and wearer comfort.

- The energy-efficient implementation of multi-antenna processing techniques guarantees wireless channel reliability at low transmit powers [9], [10].

The improvements in terms of reliability and performance will further differentiate smart textile systems from rigid portable devices. The combination of reliable, invisible, comfortable and compact integration inside a garment and stable high performance in proximity of the body with low RF-field absorption in body tissues are clear distinctive assets of SFIT technology in healthcare applications. The modularity of our active textile modules leverages deployment for a wide range of applications. We demonstrate this by considering three typical application scenarios.

In Section II, we apply SIW technology to textile antennas to develop high-performance wearable antenna modules for wireless communication via the $2.45 \mathrm{GHz} \mathrm{WiFi}$ and $4 \mathrm{G}$ LTE band 7 standards. We demonstrate how active electronics may be integrated directly underneath the antenna cavity and how energy harvesters may be placed directly on top of the antenna, without affecting the antenna performance. These features make the compact modules extremely suitable to set up mobile communication links between caregivers and a coordination centre during interventions. Section III presents a wearable wireless node with sensing and communication capabilities implemented on a dual-polarized textile antenna platform. Such nodes, unobtrusively and comfortably integrated into patient garments, are extremely useful as wireless sensor systems for patient monitoring. Finally, in Section IV we demonstrate how a dedicated wearable repeater system combined with diversity techniques may improve the wireless channel between an endoscopy capsule and a remote monitoring station such that it leverages real-time high-datarate video streaming.

\section{Substrate Integrated WaVEguide TEXTILE ANTENNAS}

For rescue workers during interventions and for patients in home care, there may be a need for a continuous reliable wireless connection to implement remote monitoring and to issue alarms, if required. For such purpose, a WiFi connection in the 


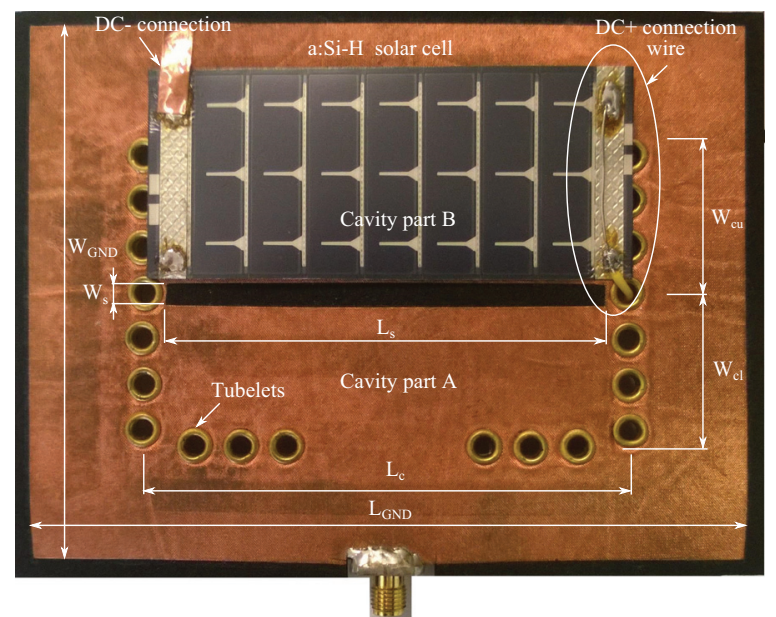

(a)

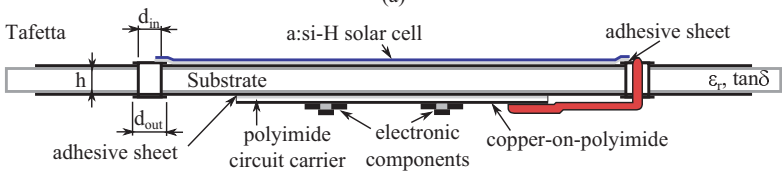

(b)

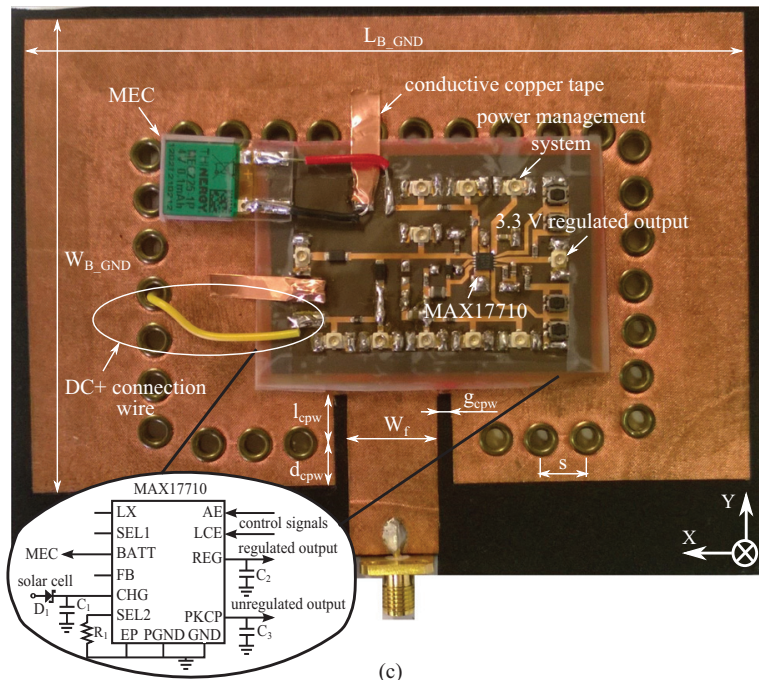

(c)

Fig. 1. Wearable cavity-backed SIW slot antenna with (a) integrated flexible solar cell on its top plane and (b) power mangement module integrated on its bottom plane.

2.45 GHz Industrial, Scientific and Medical (ISM) band may be used or, in absence of this infrastructure, a 4G Long-Term Evolution (LTE) wireless metropolitan network (WMAN) link. For this application, Fig. 1 proposes a wearable textile antenna with return loss larger than $10 \mathrm{~dB}$ in the $2.45 \mathrm{GHz}$ ISM band ([2.4-2.4835] GHz) and in the uplink and downlink of the 4G LTE band 7 ([2.50-2.57] GHz and [2.62-2.69] GHz, respectively). The SIW cavity, constructed by perforating the antenna substrate and top and bottom Flectron electro-textile sheets by rows of tubelets, in combination with a radiating slot, realizes excellent isolation of the antenna radiation from the human body. Fig. 2 demonstrates that, by exciting two modes inside the SIW cavity, robust matching remains ensured in both WiFi and 4G bands, even when placing solar cells on the cavity's top plane, together with a power management

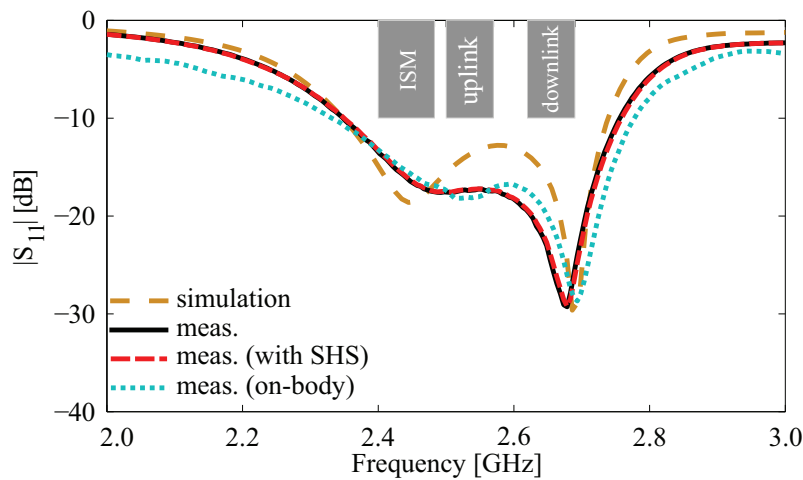

Fig. 2. Reflection coefficient of the cavity-backed SIW slot antenna with and without solar harvesting system.
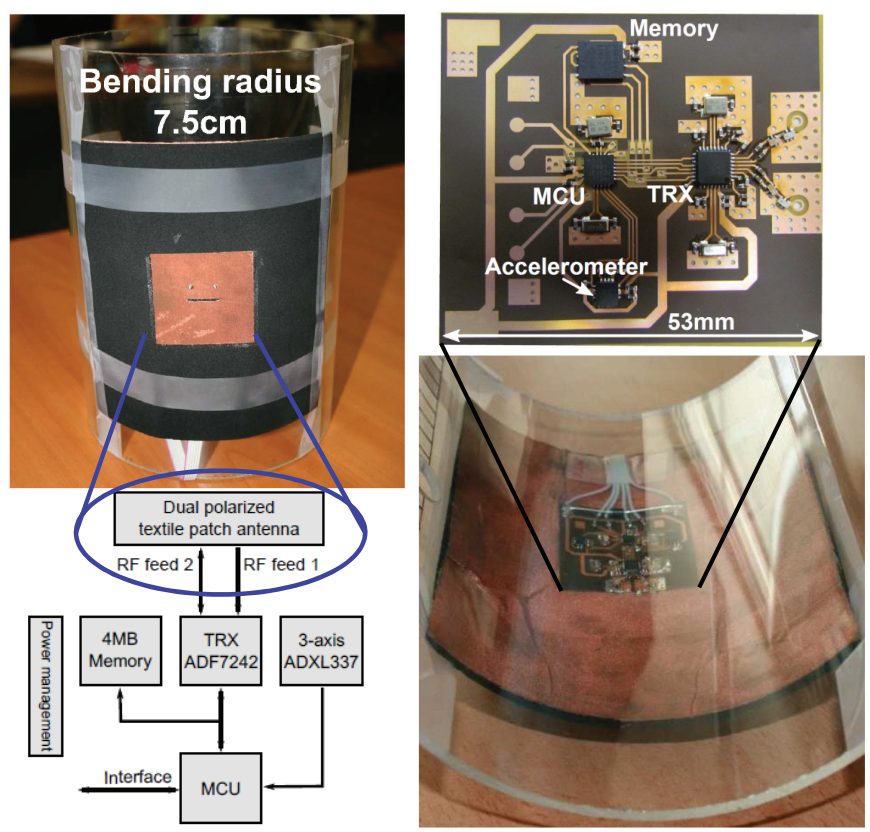

Fig. 3. Wearable wireless node implemented on a dual-polarized textile antenna (top left), consisting of a transceiver (RTX), on-board memory, microcontroller (MCU) and sensor (accelerometer).

system (PMS) on the bottom plane, and when deploying this solar antenna on a human body. By routing wires connecting the solar cells to the PMS through the vias implementing the SIW cavity, the antenna performance remains unaffected. More details about this antenna are given in [11].

\section{TEXTILE WIRELESS NODE}

An even more pervasive integration strategy may be adopted, where complete electronic circuits on an ultrathin flexible polyimide substrate are directly positioned on a textile antenna platform. Fig. 3 showcases such a wireless node, consisting of an Analog Devices ADF7242 diversity transceiver (RTX), 4MB on-board flash memory, a compact low-power Silicon Laboratories C8051F921 microcontroller (MCU) and a three-axis accelerometer, all deployed on a dual-polarized textile antenna. As seen on the figure, the wearable node is fully flexible and offers stable and reliable performance 


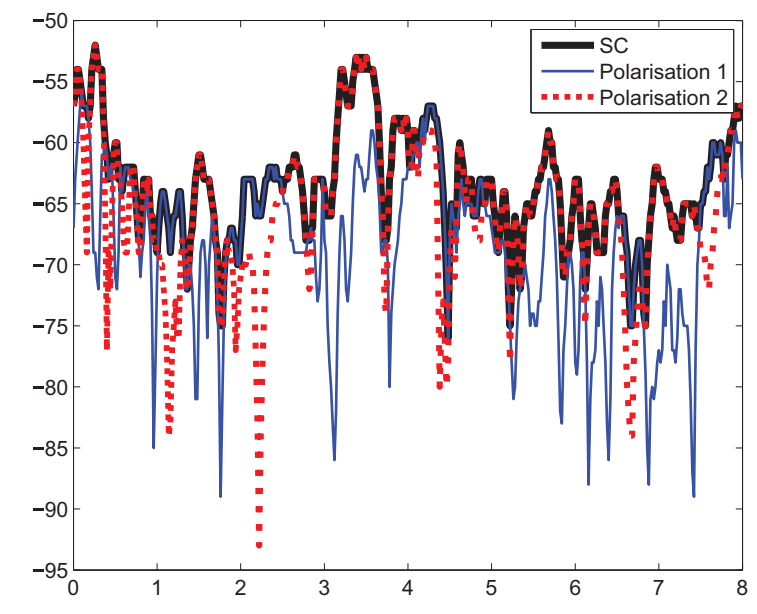

Fig. 4. Signal levels measured by the wearable wireless node deployed on a human body, at each of the antenna terminals and after selection combining.

even for bending radius of $7.5 \mathrm{~cm}$. To demonstrate the gain in wireless link reliability realized by the diversity transceiver in combination with the dual-polarized antenna, we show in Fig. 4 the signal levels measured at each antenna terminal as well as the signal resulting from a selection combiner. These levels were measured and recorded in the on-board memory while the node was deployed on a human body and operating autonomously, without any cables attached. For more details we refer to [12].

\section{WEARABLE REPEATER SYSTEM FOR WIRELESS ENDOSCOPY CAPSULES}

Wireless communication links with implanted devices suffer from very large path losses due to RF-field attenuation by the human body. This makes it extremely difficult to guarantee the signal-to-noise ratios needed for high-datarate communication, as required for real-time wireless endoscopy applications. We propose a distributed wearable multi-antenna system [13] that overcomes this problem by combining an innovative textile repeater antenna design with multi-antenna diversity combining. Fig. 5 shows the distributed diversity system consisting of eight textile repeater antennas. In contrast to previous sections, the antenna, depicted at the top right of Fig. 5, is designed for optimal antenna performance when oriented towards the body, operating as a receive antenna for signals transmitted by wireless endoscopy capsules. To ensure stable radiation characteristics when deployed at different areas of the body, a spacer superstrate is applied on top of the antenna patch. The $8^{\text {th }}$-order antenna diversity system ensured a sufficiently high Signal-t-Noise Ratio (SNR) for real-time high-datarate wireless endoscopy, as illustrated in Fig. 6, where we see that more than $20 \mathrm{~dB}$ SNR is achieved for all positions. By simply adding an analog amplifier, providing $43.8 \mathrm{~dB}$ of gain at $2.45 \mathrm{GHz}$ and an off-body textile transmit antenna [14], the wireless endoscopy capsule data may be relayed to a remote access point, as shown in the experimental setup in Fig. 7. In this configuration [15], where we focus on a single repeater antenna acting as relay, we assess the data

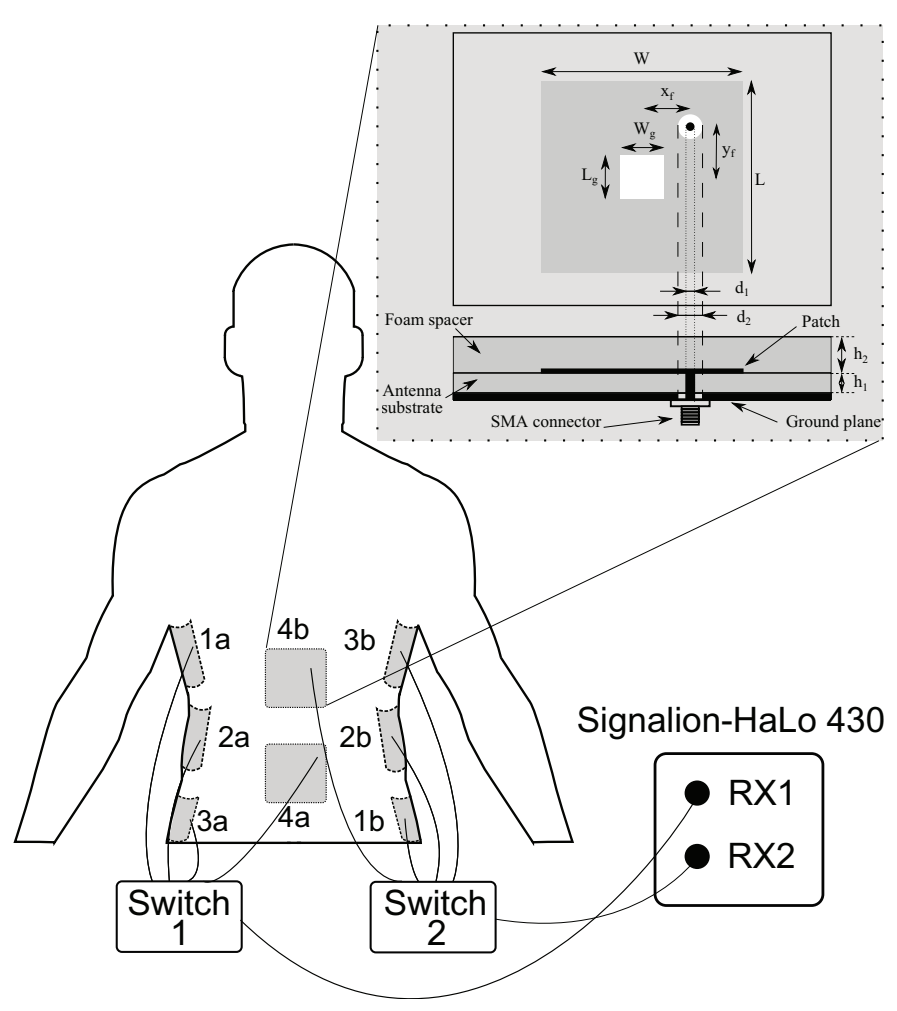

Fig. 5. Wearable textile repeater antenna (top right) deployed in a diversity configuration for wireless endoscopy. For measurement purposes, all eight antennas are connected to a Signalion Halo 430 testbed.

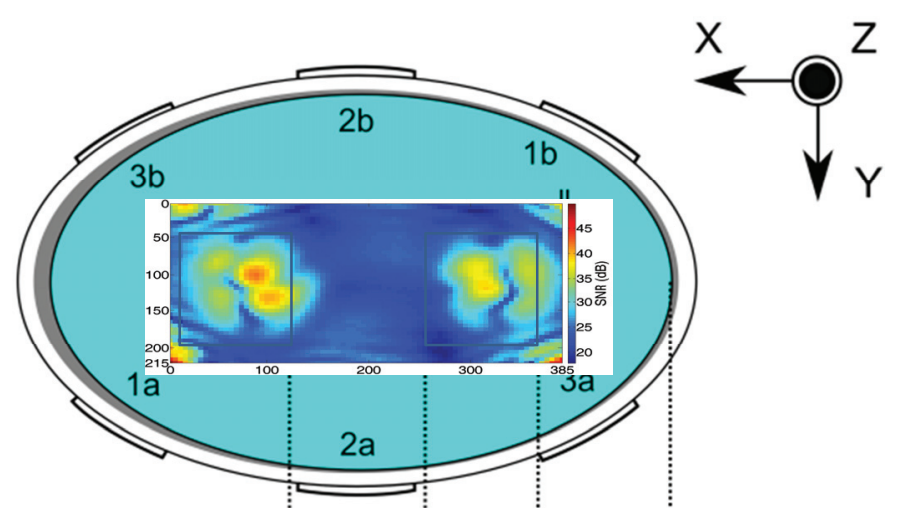

Fig. 6. SNR (dB) measured by the $8^{t h}$-order antenna diversity system as a function of the position of the $x$-oriented insulated implanted dipole at a depth of $4.3 \mathrm{~cm}$ underneath the liquid surface and $5.9 \mathrm{~cm}$ away from the bottom. Dimensions on the field plot are in $\mathrm{mm}$.

link quality between an implanted transmit antenna, placed at various depths in an ELI flat phantom, filled with tissuemimicking fluid MSL2450 mimicking the human body torse, and a receiver. An implantable dipole antenna moves along implant positions, varying from 10 to $10 \mathrm{~cm}$ along the $\mathrm{x}$-axis, relative to the repeaters position (Fig. 7), and depths, ranging from 1 to $10 \mathrm{~cm}$ along the $\mathrm{y}$-axis. The SNR measured by the textile receive antenna deployed on the phantom is shown in the left subfigure of Fig. 8. An average SNR of $41 \mathrm{~dB}$ is observed, while the SNR exceeds $15 \mathrm{~dB}$ for depths up to $85 \mathrm{~mm}$ in the body. The repeater then amplifies and relays 


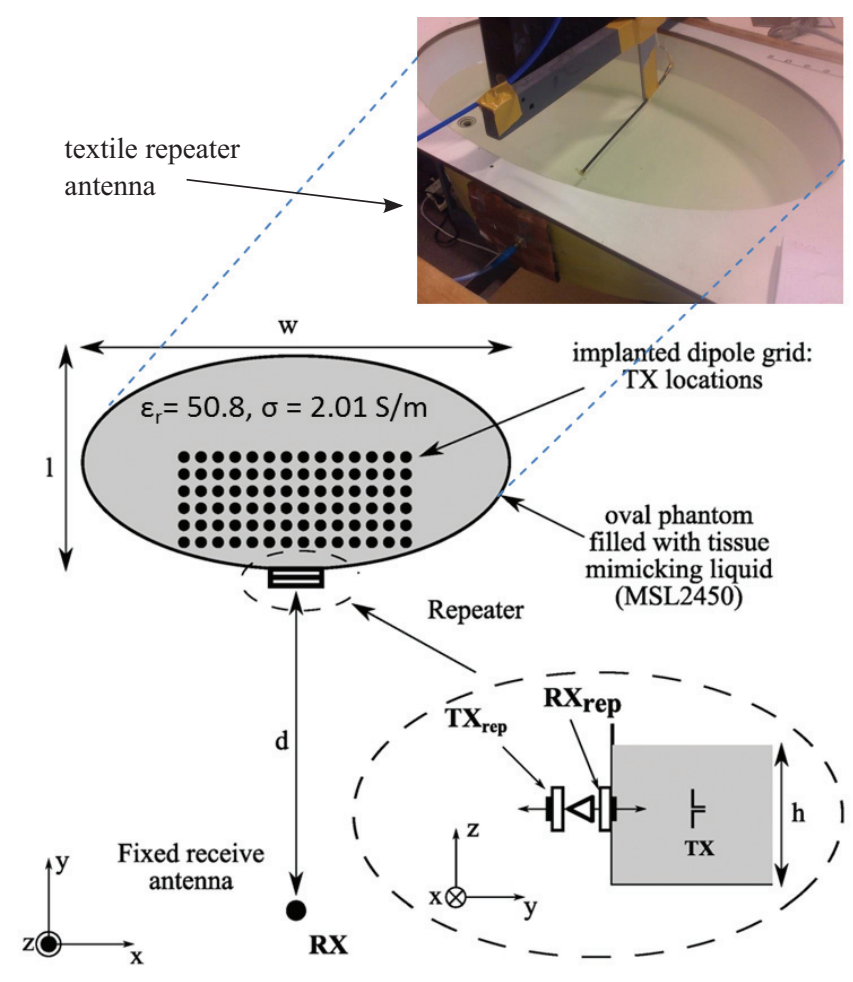

Fig. 7. Wearable textile repeater antenna relaying wireless endoscopy data to a remote access point. For experimental validation, the antenna is placed on an ELI flat phantom filled with MSL2450 tissue-mimicking liquid.
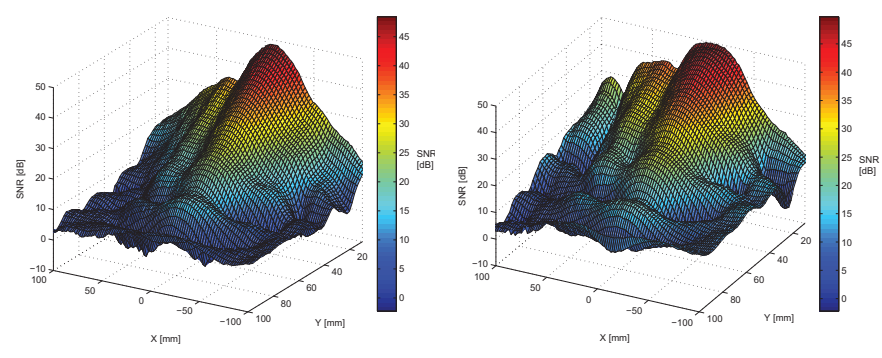

Fig. 8. Measured SNR by textile receive antenna on the phantom (left) and by external antenna (right), after the signal is relayed and amplified by the textile repeater antenna.

these received data to a remote access point at $2 \mathrm{~m}$ distance. The resulting SNR received by this remote antenna is shown in the right subfigure of Fig. 8. As a result, the SNR values observed at the remote access point are very comparable to the ones measured by the repeater antenna, which represents a tremendous improvement compared to the average SNR of $11 \mathrm{~dB}$ for the same setup without on-body repeater system.

\section{CONCLUSION}

Whereas in the past wearable electronics, and textile antennas in particular, were mainly of interest from a research perspective, by now they have reached the levels of maturity and reliabilty needed for commercial application. In this paper, we discuss some recent developments that make use of substrate integrated waveguide technology and pervasive integration of electronics and energy harvesters on the antenna platform to come to wireless nodes that have the potential of being applicable in healthcare applications.

\section{ACKNOWLEDGMENT}

This research was partially funded by the Inter-University Attraction Poles Program initiated by the Belgian Science Policy Office.

\section{REFERENCES}

[1] J. Lilja, P. Salonen, T. Kaija, and P. de Maagt, "Design and Manufacturing of Robust Textile Antennas for Harsh Environments," IEEE TRANSACTIONS ON ANTENNAS AND PROPAGATION, vol. 60, no. 9, pp. 4130-4140, SEP 2012.

[2] M. L. Scarpello, I. Kazani, C. Hertleer, H. Rogier, and D. Vande Ginste, "Stability and Efficiency of Screen-Printed Wearable and Washable Antennas," IEEE ANTENNAS AND WIRELESS PROPAGATION LETTERS, vol. 11, pp. 838-841, 2012.

[3] F. Declercq, I. Couckuyt, H. Rogier, and T. Dhaene, "Environmental High Frequency Characterization of Fabrics Based on a Novel Surrogate Modelling Antenna Technique," IEEE TRANSACTIONS ON ANTENNAS AND PROPAGATION, vol. 61, no. 10, pp. 5200-5213, OCT 2013.

[4] R. Moro, S. Agneessens, H. Rogier, and M. Bozzi, "Wearable textile antenna in substrate integrated waveguide technology," ELECTRONICS LETTERS, vol. 48, no. 16, pp. 985-986, AUG 22012.

[5] T. Kaufmann and C. Fumeaux, "Wearable Textile Half-Mode SubstrateIntegrated Cavity Antenna Using Embroidered Vias," IEEE ANTENNAS AND WIRELESS PROPAGATION LETTERS, vol. 12, pp. 805-808, 2013.

[6] S. Agneessens and H. Rogier, "Compact half diamond dual-band textile HMSIW on-body antenna," Antennas and Propagation, IEEE Transactions on, vol. 62, no. 5, pp. 2374-2381, May 2014.

[7] A. Dierck, H. Rogier, and F. Declercq, "A Wearable Active Antenna for Global Positioning System and Satellite Phone," IEEE TRANSACTIONS ON ANTENNAS AND PROPAGATION, vol. 61, no. 2, pp. 532-538, FEB 2013.

[8] F. Declercq and H. Rogier, "Active Integrated Wearable Textile Antenna With Optimized Noise Characteristics," IEEE TRANSACTIONS ON ANTENNAS AND PROPAGATION, vol. 58, no. 9, pp. 3050-3054, SEP 2010.

[9] P. Van Torre, M. L. Scarpello, L. Vallozzi, H. Rogier, M. Moeneclaey, D. Vande Ginste, and J. Verhaevert, "Indoor Off-Body Wireless Communication: Static Beamforming versus Space-Time Coding," INTERNATIONAL JOURNAL OF ANTENNAS AND PROPAGATION, 2012.

[10] P. Van Torre, L. Vallozzi, C. Hertleer, H. Rogier, M. Moeneclaey, and J. Verhaevert, "Indoor Off-Body Wireless MIMO Communication With Dual Polarized Textile Antennas," IEEE TRANSACTIONS ON ANTENNAS AND PROPAGATION, vol. 59, no. 2, pp. 631-642, FEB 2011.

[11] S. Lemey, F. Declercq, and H. Rogier, "Dual-Band Substrate Integrated Waveguide Textile Antenna With Integrated Solar Harvester," IEEE ANTENNAS AND WIRELESS PROPAGATION LETTERS, vol. 13, pp. 269-272, 2014.

[12] P. Vanveerdeghem, P. Van Torre, C. Stevens, J. Knockaert, and H. Rogier, "Flexible dual-diversity wearable wireless node integrated on a dual-polarized textile patch," IET SCIENCE MEASUREMENT \& TECHNOLOGY, DOI: 10.1049/iet-smt.2013.0224, 2014.

[13] T. Castel, P. Van Torre, E. Tanghe, S. Agneessens, G. Vermeeren, W. Joseph, and H. Rogier, "Improved Reception of In-Body Signals by Means of a Wearable Multi-Antenna System," INTERNATIONAL JOURNAL OF ANTENNAS AND PROPAGATION, 2013.

[14] L. Vallozzi, P. Van Torre, C. Hertleer, H. Rogier, M. Moeneclaey, and J. Verhaevert, "Wireless Communication for Firefighters Using Dual-Polarized Textile Antennas Integrated in Their Garment," IEEE TRANSACTIONS ON ANTENNAS AND PROPAGATION, vol. 58, no. 4, pp. 1357-1368, APR 2010.

[15] S. Agneessens, P. Van Torre, E. Tanghe, G. Vermeeren, W. Joseph, and H. Rogier, "On-Body Wearable Repeater as a Data Link Relay for In-Body Wireless Implants," IEEE ANTENNAS AND WIRELESS PROPAGATION LETTERS, vol. 11, pp. 1714-1717, 2012. 